\title{
Matrix metalloproteinases 2 and 9 work in concert to produce aortic aneurysms
}

\author{
G. Matthew Longo, ${ }^{1}$ Wanfen Xiong, ${ }^{1}$ Timothy C. Greiner ${ }^{2}$ Yong Zhao, ${ }^{1}$ Nicola Fiotti, ${ }^{1}$ \\ and B. Timothy Baxter ${ }^{1,3}$
}

${ }^{1}$ Department of Surgery,

${ }^{2}$ Department of Pathology and Microbiology, and

${ }^{3}$ Department of Cell Biology and Anatomy, University of Nebraska Medical Center, Omaha, Nebraska, USA

\begin{abstract}
Matrix metalloproteinases (MMPs) 9 and 2 are increased in human abdominal aortic aneurysm (AAA) tissue, but their precise role and potential interaction remain unclear. Experimental induction of aortic aneurysms in mice genetically deficient in these peptidases could provide new insight into AAA pathogenesis. Mice deficient in the expression of MMP-9 (MMP-9KO) or MMP-2 (MMP-2KO) and their corresponding wild-type background mice (WT) underwent AAA induction by abluminal application of calcium chloride $\left(\mathrm{CaCl}_{2}\right)$. No aneurysm formation was observed at 10 weeks after treatment in either the MMP-9KO or the MMP-2KO mice, whereas the corresponding WT mice showed an average $74 \%$ and $52 \%$ increase in aortic diameter, respectively. Reinfusion of competent macrophages from the corresponding WT strains into knockout mice resulted in reconstitution of AAA in MMP-9KO but not MMP-2KO mice. These findings suggest that macrophage-derived MMP-9 and mesenchymal cell MMP-2 are both required and work in concert to produce AAA.
\end{abstract}

J. Clin. Invest. 110:625-632 (2002). doi:10.1172/JCI200215334.

\section{Introduction}

Abdominal aortic aneurysm (AAA) is a common and lethal disorder $(1,2)$. Through studies of human tissue and animal models of AAA, we have begun to understand the underlying pathophysiology. These studies have led to a paradigm shift in our concepts about AAA. Rather than a simple degenerative process, AAA has proven to be a dynamic remodeling process.

Inflammation, which is temporally and spatially associated with disruption of the orderly lamellar structure of the aortic media, appears to play a fundamental role in AAA development and progression. Studies of human AAA tissue have identified extensive inflammatory infiltrates in both the media and adventitia. Macrophages and $\mathrm{T}$ lymphocytes are the predominant immune subsets $(3,4)$. Two widely used experimental models of AAA rely on local induction of an intense inflammatory response $(5,6)$, which precedes a period of gradual aortic dilatation. Progressive destruction of

Received for publication February 25, 2002, and accepted in revised form July 9, 2002.

Address correspondence to: B. Timothy Baxter, Surgery Research Department, LTC Room 9727, University of Nebraska Medical Center, 600 South 42nd Street, Omaha, Nebraska 68198-7690, USA. Phone: (402) 559-7193; Fax: (402) 559-6732; E-mail: btbaxter@unmc.edu.

G. Matthew Longo and Wanfen Xiong contributed equally to this work.

Conflict of interest: No conflict of interest has been declared. Nonstandard abbreviations used: abdominal aortic aneurysm (AAA); matrix metalloproteinase (MMP); knockout (KO); wildtype (WT); hematoxylin and eosin (H\&E); 5-(and-6)carboxyfluorescein diacetate, succinimidyl ester (CFSE). the normal lamellar architecture with flattening and fragmentation of elastin fibers accompanies aortic expansion. Attenuation of the inflammatory response in animal models inhibits dilatation, while augmenting the response increases the frequency and size of the aneurysms $(7,8)$. This breakdown of normally longlived matrix macromolecules such as elastin has been ascribed to a family of endopeptidases, the matrix metalloproteinases (MMPs).

Increased expression of MMPs (8-11) has been observed in human aneurysm tissue. The prominent elastin degradation has led to the investigation of the MMPs with elastolytic capabilities. Both the mRNA and protein levels of MMP-2 are increased in human AAA tissues compared with normal aorta. An increased proportion of the MMP-2 in AAA tissue is found in the activated form, and there is indirect evidence of increased matrix binding of activated MMP-2 (11). Immunohistochemical studies colocalize MMP-2 with mesenchymal cells (smooth muscle cells and fibroblasts) and, less commonly, macrophages (11). MMP-9 is the most abundant gelatinolytic MMP in AAA tissue, and it is secreted in high levels from AAA explants (9). Since MMP-9 and, to a lesser extent, MMP-2 are products associated with macrophage invasion, their presence in AAA is expected and does not demonstrate a causal role, nor does it preclude an important role for other MMPs, serine or cysteine proteases. Several lines of investigation using animal models of AAA have shown, however, that MMPs have a pivotal role in this process (12-14).

Using a model of arterial aneurysm described first by Gertz et al. (6) in the rabbit carotid artery and subse- 
quently by Freestone et al. (8) in the rabbit aorta, we have developed a murine model of AAA. This model recapitulates three of the key features of human aneurysms: intense local inflammation, increased expression of MMP-2 and MMP-9, and local matrix destruction (15). We have investigated the role of MMP-2 and MMP-9 in this model using mice with targeted deletions of these MMPs. We find that both the MMP-9 and the MMP-2 knockout (MMP-9KO and MMP-2KO) mice are resistant to aneurysm formation. This is not related to the inability of the macrophages to invade the aortic tissue. Aneurysms can be reconstituted in the MMP-9KO mice by intravenous infusion of wild-type (WT) macrophages. Infusion of competent WT macrophages into MMP-2KO mice did not reconstitute the aneurysms. This failure of the WT macrophages to reconstitute the aneurysm suggests that the important contribution of MMP- 2 to AAA is not macrophage-derived but is, more likely, from mesenchymal cells. Macrophage-derived MMP-9 and mesenchymal cell-derived MMP-2 work in concert to produce aneurysms in our murine model. The protection of the matrix and lack of aneurysm formation in the absence of either MMP-2 or MMP-9 are intriguing and suggest requirement of an interaction between the proteolytic products of the invading inflammatory cells and local mesenchymal cells.

\section{Methods}

Mice. The homozygous MMP-9KO mice were obtained from Robert Thompson (Washington University, St. Louis, Missouri, USA) and have been described in recent publications (14). The MMP-9KO animals are bred on a $129 / \mathrm{SvEv}$ background and are genetically identical to the WT 129/SvEv animals. The 129/SvEv mice were purchased from Taconic (Germantown, New York, USA). The homozygous MMP-2KO mice were first described by Itoh et al. (16). They were obtained from David Muir (University of Florida, Gainesville, Florida, USA). These MMP-2KO mice have been backcrossed into C57BL/ 6 background eight times. The WT controls for the MMP-2KO mice, C57BL/6 mice, were purchased from The Jackson Laboratory (Bar Harbor, Maine, USA). Knockout colonies were genotyped by the laboratories that provided them, and the absence of expression of MMP-9 or MMP-2 was confirmed by zymography of tail and aortic tissue.

Aneurysm induction model. Mice were anesthetized and underwent laparotomy at age 8 weeks. The abdominal aorta between the renal arteries and bifurcation of the iliac arteries was isolated from the surrounding retroperitoneal structures. The diameter of the aorta was measured in triplicate midway between the renal artery origin and iliac artery bifurcation using a videomicrometer from Colorado Video Inc. (Boulder, Colorado, USA). After base-line measurements, $0.25 \mathrm{M}$ $\mathrm{CaCl}_{2}$ was applied to the external surface of the aorta. $\mathrm{NaCl}(0.9 \%)$ was substituted for $\mathrm{CaCl}_{2}$ in sham control mice. After 15 minutes the aorta was rinsed with $0.9 \%$
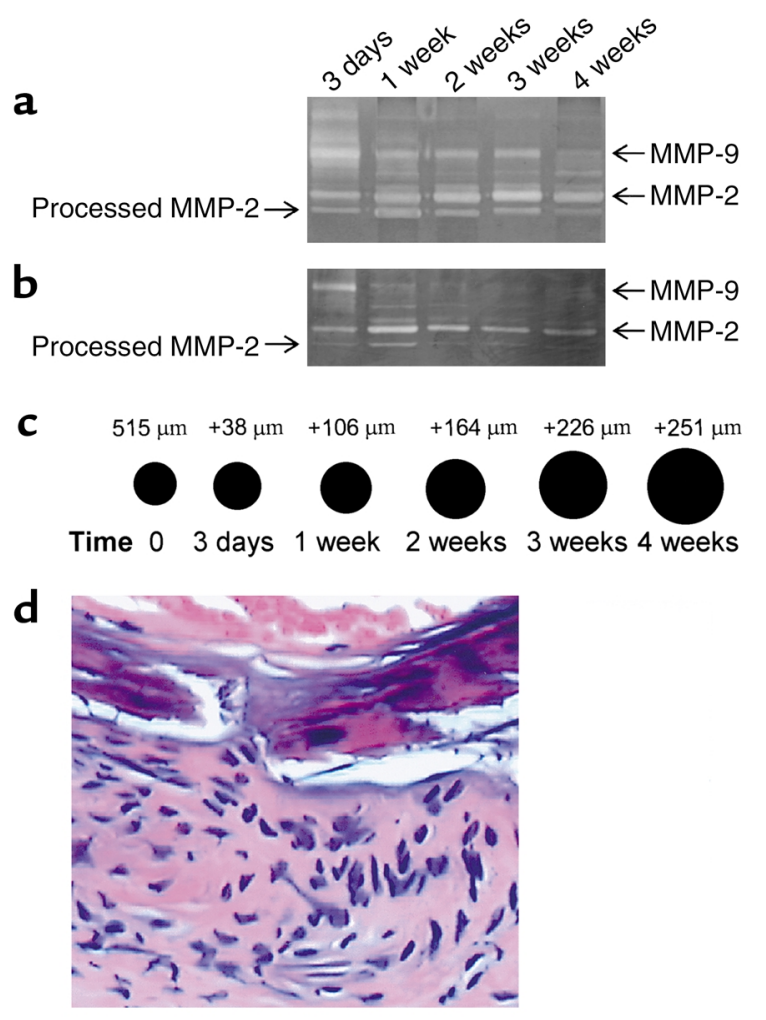

\section{Figure 1}

The time course of expression of MMP-9 and MMP-2 and corresponding aneurysm growth. WT mouse aortae were incubated with $\mathrm{CaCl}_{2}$ or $\mathrm{NaCl}$. Three days, 1 week, and 2, 3, and 4 weeks after treatment, the aortic diameters were measured and mice were sacrificed. (a) $\mathrm{CaCl}_{2}$-treated mice; (b) $\mathrm{NaCl}$-treated mice (control). (c) Aortic diameter increases in $\mathrm{CaCl}_{2}$-treated mice at indicated time points. (d) $\mathrm{H} \& \mathrm{E}$ staining of $\mathrm{CaCl}_{2}$-treated mouse aortic section, showing a significant inflammatory infiltrate at 3 days.

sterile saline and the incision was closed; mice were returned to their cages after recovery. Ten weeks later the mice underwent laparotomy and dissection. Measurements were repeated at the same location in the midinfrarenal aorta. The measurements were made by an observer unaware of the initial treatment $(\mathrm{NaCl}$ or $\mathrm{CaCl}_{2}$ ). Typically, there was diffuse, homogeneous dilatation of the infrarenal aorta. The aorta was collected for zymographic analysis of MMP proteins. For histologic studies, the aorta was perfusion-fixed with $10 \%$ neutral-buffered formalin.

Gelatin zymography. Aortic proteins and peritoneal macrophage cellular proteins were extracted as previously described (11). Samples standardized for protein concentration were resolved by nondenaturing electrophoresis through a $10 \%$ SDS-PAGE copolymerized with $0.8 \%$ gelatin. The molecular sizes of gelatinolytic activities were determined using protein standards (Bio-Rad Laboratories Inc., Hercules, California, USA). A series of animals were sacrificed at earlier time intervals ( 3 days to 4 weeks) to examine the temporal expression of MMP-9 and MMP-2 relative to the increase in aortic diameter (Figure 1, $a$ and $b$ ). 
Histology and microscopy. For hematoxylin-and-eosin (H\&E) staining, after perfusion-fixation with $10 \%$ neutral-buffered formalin, abdominal aortic tissues were embedded in paraffin and cut into $4-\mu \mathrm{m}$ sections. The sections were stained with H\&E. Figure $1 \mathrm{~d}$ shows the inflammatory infiltrate observed 3 days after $\mathrm{CaCl}_{2}$ aneurysm induction. Movat's pentachrome staining on paraffin-blocked specimens was carried out according to the method described by Russell (17). For Masson's Trichrome staining, the slides were stained with hematoxylin, crocein scarlet, acid fuchsin, and aniline blue (Sigma-Aldrich, St. Louis, Missouri, USA). Each staining cycle alternated between fixing and washing procedures. The slides were examined and photographed using light microscopy (Boyce Scientific Inc., Gray Summit, Missouri, USA) $(\times 20)$.

Immunohistochemistry. Mice underwent AAA induction according to the method described above. Three mice in each group were sacrificed at 1,2, and 10 weeks for macrophage staining performed on paraffin-embedded 4- $\mu \mathrm{m}$ aortic sections. The sections were incubated with a monoclonal rat anti-mouse Mac3 antibody (PharMingen, San Diego, California, USA) diluted 1:500 for 30 minutes at $37^{\circ} \mathrm{C}$. The sections were then briefly washed in citrate solution and subsequently incubated with the secondary antibody, which is a mouse-absorbed, biotin-conjugated rabbit anti-rat IgG. Macrophage staining was examined using light microscopy. Positive controls and nonimmune negative controls were performed. Macrophage infiltration was graded in the aortic media and adventitia by a pathologist unaware of the genotype or time of sacrifice. Four separate sections from each aorta were stained and evaluated, and the mean grade was reported.

Isolation and infusion of peritoneal macrophage. 129/SvEv, C57BL/6, and MMP-9KO mice were injected intraperitoneally with $1 \mathrm{ml}$ of $3 \%$ Brewer thioglycollate medium. A week later, peritoneal macrophages were collected, washed, and resuspended in PBS. Cells were labeled with 5-(and 6)-carboxyfluorescein diacetate, succinimidyl ester (CFSE) (Molecular Probes Inc., Eugene, Oregon, USA). Groups of six to eight MMP-2KO and MMP-9KO mice were injected with $5 \times 10^{7}$ peritoneal macrophages via the tail vein 1 day before AAA induction. A second macrophage infusion was repeated a week later. Before the second infusion, a blood sample was obtained to quantify residual CFSE-labeled macrophages using FACScan. To determine the number of CFSE-labeled macrophages in the aneurysm, three animals were sacrificed 1 week after infusion. Aortic cells were isolated as described previously (11), and CFSE-labeled macrophages were quantified using FACScan.

Statistical analysis. Measurements of aortic diameter are expressed as mean value $\pm S E$. A paired Student's $t$ test was used to compare original and final diameter. Statistical significance was accepted at $P<0.05$. Analysis of the macrophage infiltration of the media and adventitia was evaluated with a Mann-Whitney test, taking into account the Bonferroni correction. Statistical significance was taken at $P<0.05$ when the Bonferroni correction was carried out.

\section{Results}

Induction of AAA in WT, MMP-2KO, and MMP-9KO mice. Increased expression of MMP-2 has been observed in AAA tissue $(11,18)$. Thus, MMP- 2 may be required for AAA formation. To test this hypothesis, we examined the response of $\mathrm{MMP}-2 \mathrm{KO}$ mice to $\mathrm{CaCl}_{2}$ aneurysmal induction. Ten weeks after periaortic application of $\mathrm{CaCl}_{2}$, there was no significant change in aortic diameter or histology in MMP-2KO mice (Table 1; Figure 2, a and $\mathrm{f}$ ), which were similar to the $\mathrm{NaCl}$-treated group (Figure 2e). However, C57BL/6 WT mice showed a $52.3 \% \pm 4.5 \%$ increase in aortic diameter after $\mathrm{CaCl}_{2}$ treatment (Table 1; Figure 2a). Trichrome staining of aortic sections from these mice showed disruption and fragmentation of medial elastic lamellae (Figure 2d).

To examine the role of MMP-9 in AAA development, we performed $\mathrm{CaCl}_{2}$ aneurysm induction on MMP$9 \mathrm{KO}$ mice. There was no significant increase in the aortic diameter, similar to the NaCl-treated controls (Table 2). The 129/SvEv mice consistently developed aneurysms showing a $74.0 \% \pm 12.3 \%$ increase in diameter. Figure $2 \mathrm{~b}$ illustrates the initial and final measurements of aorta. Connective tissue staining of aortic sections from $\mathrm{CaCl}_{2}$-treated MMP-9KO mice did not show any detectable damage to the medial elastic lamellae (Figure $2 \mathrm{j}$ ). The aortic wall appeared markedly similar to that of the NaCl-treated MMP-9KO control mice (Figure 2, g and i). The $\mathrm{CaCl}_{2}$-treated WT mice exhibited flattening and destruction of the elastic lamellae (Figure 2h). Fragmented elastic fibers were observed around macrophages in $\mathrm{CaCl}_{2}$-induced WT mouse aneurysm tissues (Figure 2k). Although macrophages were also present in $\mathrm{CaCl}_{2}$-treated MMP$9 \mathrm{KO}$ (Figure 21) and MMP-2KO (Figure $2 \mathrm{~m}$ ) mouse aortae, the adjacent elastic fibers were intact. These observations suggest that the aneurysm inhibitory effects seen in the MMP-9KO and MMP-2KO mice

\section{Table 1}

Changes in aortic diameter in WT and MMP-2KO mice after treatment with $\mathrm{NaCl}$ and $\mathrm{CaCl}_{2}$

\begin{tabular}{lcccc}
\hline & \multicolumn{2}{c}{ C57BL/6 } & \multicolumn{2}{c}{ MMP-2KO } \\
Treatment & $\mathrm{NaCl}$ & $\mathrm{CaCl}_{2}$ & $\mathrm{NaCl}$ & $\mathrm{CaCl}_{2}$ \\
Number & 10 & 10 & 5 & 5 \\
Pretreatment $(\mu \mathrm{m})$ & $538 \pm 8.2$ & $515 \pm 6.7$ & $527 \pm 12.1$ & $495 \pm 13.0$ \\
Post-treatment $(\mu \mathrm{m})$ & $548 \pm 4.0$ & $784 \pm 9.2^{\mathrm{A}}$ & $553 \pm 13.2$ & $535 \pm 7.6$ \\
AAA development $(\%)$ & 0 & 60 & 0 & 0 \\
Percent increase & 3 & 52.3 & 5 & 8 \\
Range of increase $(\%)$ & $0-16$ & $43-70$ & $4-6$ & $3-14$ \\
\hline
\end{tabular}

Aortic diameters were measured before $\mathrm{NaCl}$ (or $\mathrm{CaCl}_{2}$ ) incubation (pretreatment) and at sacrifice (post-treatment). Measurements of aortic diameter are expressed as mean \pm SE. The percent increase is represented as compared with pretreatment. The development of aneurysm was defined as an increase $\geq 50 \%$ relative to the original diameter of the aorta. ${ }^{A} P<0.01$, Student's $t$ test, compared with pretreatment. 

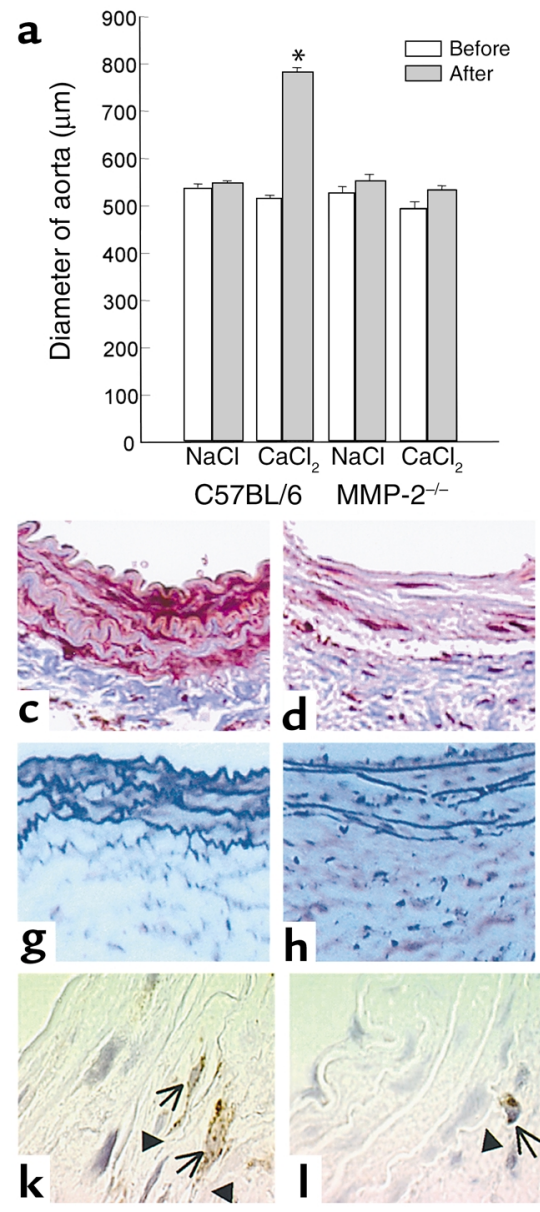

b
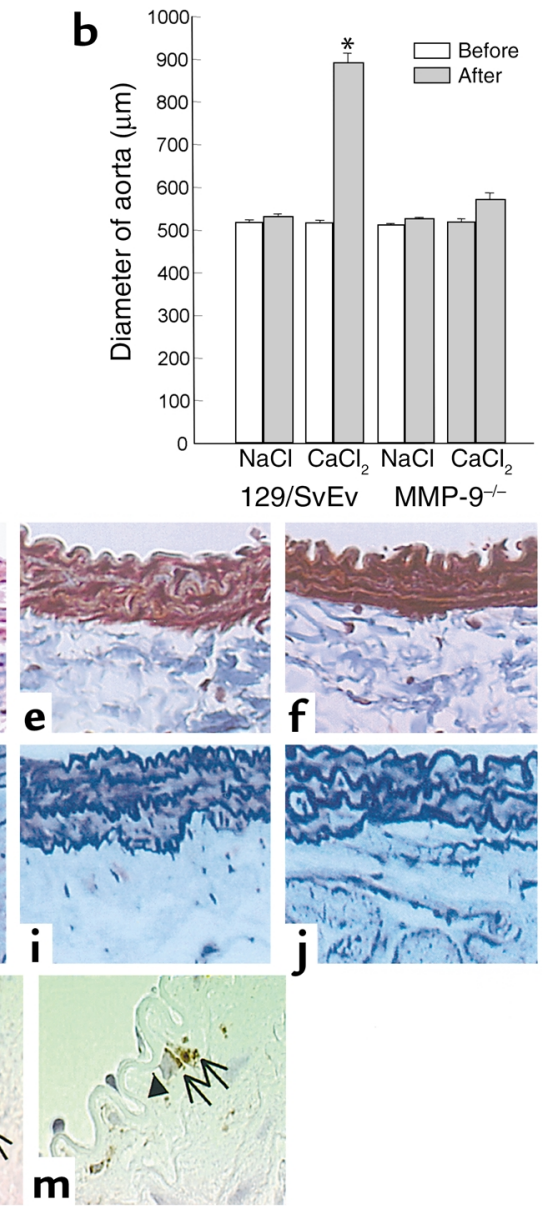

Figure 2

(a and b) Aortic diameter changes after $\mathrm{NaCl}$ and $\mathrm{CaCl}_{2}$ treatment in $\mathrm{C} 57 \mathrm{BL} / 6$ and MMP-2KO mice (a), and in 129/SvEv and MMP-9KO mice (b). Aortic diameters were measured before $\mathrm{NaCl}$ or $\mathrm{CaCl}_{2}$ incubation (white bars) and at sacrifice (gray bars). Bars shown in each group represent the mean \pm SE of 5-14 mice. The diameter in the $\mathrm{CaCl}_{2}$-treated group was increased significantly at sacrifice in C57BL/ 6 and 129/SvEv mice $\left({ }^{*} \mathrm{P}<0.01\right)$. (c-m) Histologic changes of mouse aorta at 10 weeks after treatment. (c-f) Trichrome staining for elastic fibers. $\mathrm{NaCl}$-treated (c) and $\mathrm{CaCl}_{2}$-treated (d) aorta from $\mathrm{C} 57 \mathrm{BL} / 6$ and $\mathrm{NaCl}$-treated (e) or $\mathrm{CaCl}_{2}$-treated (f) aorta from MMP-2KO mice. (g-j) Pentachrome staining for elastic fibers. $\mathrm{NaCl}$-treated $(\mathbf{g})$ and $\mathrm{CaCl}_{2}-$ treated (h) aorta from 129/SvEv and $\mathrm{NaCl}$-treated (i) or $\mathrm{CaCl}_{2}$-treated (j) aorta from MMP-9KO mice. (k-m) Immunoperoxidase staining for macrophages of mouse aorta 2 weeks after $\mathrm{CaCl}_{2}$ incubation in 129/SvEv (k), MMP-9KO (I), and MMP-2KO (m). Mac3-positive cells are indicated by arrows. Elastic fibers are represented by arrowheads. $(\times 100)$. were not a result of the inability of the macrophages to reach the site of injury. Instead, the aneurysm resistance in the $\mathrm{KO}$ mice appears to relate to the inability of invading macrophages to degrade surrounding matrix. Expression of MMP-2 and MMP-9 in WT, MMP- $2 K O$, and $M M P-9 K O$ mouse aorta after aneurysm induction. Aortic protein prepared from 129/SvEv, MMP-9KO, and MMP$2 \mathrm{KO}$ mice was analyzed by gelatin zymography (Figure 3). As expected, no MMP-2 and MMP-9 activity was detected in MMP-2KO and MMP-9KO mice, respectively. WT mice expressed processed MMP-2 and MMP-9 as well as pro-MMP-2 and -MMP-9. The zymograms suggest that MMP-2KO and MMP-9KO mice may have higher levels of the processed forms of MMP-9 and MMP-2, respectively, compared with WT mice. This may represent a compensatory increase in the physiologic activators of these MMPs in the knockout mice. Despite this increase in the processed form of the MMPs, the aortic matrix was protected in the absence of either MMP-2 or MMP-9.

Macrophage infiltrate in $\mathrm{CaCl}_{2}$-treated aortae. One of the remarkable histologic changes in $\mathrm{CaCl}_{2}$-treated aortae was the early inflammatory infiltrate in the adventitia and media (Figure 1d). In consideration of the possible effects of MMP-9 on macrophage migration into AAA tissues and of the observations of Ducharme et al. (19), we examined the aortic tissue for the presence of Mac3-positive macrophage cells. The numbers of macrophages in the adventitia and media were quantified 1,2, and 10 weeks after aneurysm induction (Table 3). There was no significant difference in the numbers of Mac3-positive cells in the aorta between $\mathrm{CaCl}_{2}$-treated 129/SvEv mice and MMP-9KO mice. We also confirmed the recent observation of Corry et al. (20) in demonstrating that macrophage migration into the adventitia ( 1 week, $2.6 \pm 0.3 ; 2$ weeks,

\section{Table 2}

Changes in aortic diameter in WT and MMP-9KO mice after treatment with $\mathrm{NaCl}$ and $\mathrm{CaCl}_{2}$

\begin{tabular}{lcccc}
\hline & \multicolumn{2}{c}{$129 / \mathrm{SvEv}$} & \multicolumn{2}{c}{ MMP-9KO } \\
Treatment & $\mathrm{NaCl}$ & $\mathrm{CaCl}_{2}$ & $\mathrm{NaCl}$ & $\mathrm{CaCl}_{2}$ \\
Number & 12 & 14 & 14 & 14 \\
Pretreatment $(\mu \mathrm{m})$ & $514 \pm 5.7$ & $514 \pm 5.5$ & $511 \pm 2.5$ & $514 \pm 7.6$ \\
Post-treatment $(\mu \mathrm{m})$ & $532 \pm 5.4$ & $922 \pm 21.6^{\mathrm{A}}$ & $527 \pm 2.2$ & $579 \pm 14.3$ \\
AAA development $(\%)$ & 0 & 93 & 0 & 0 \\
Percent increase & 1 & 74 & 3 & 8 \\
Range of increase $(\%)$ & $0-17$ & $36-100$ & $0-6$ & $0-32$
\end{tabular}

Aortic diameters were measured before $\mathrm{NaCl}\left(\right.$ or $\left.\mathrm{CaCl}_{2}\right)$ incubation (Pretreatment) and at sacrifice (Post-treatment). Measurements of aortic diameter are expressed as mean \pm SE. The percent increase is represented as compared with pretreatment. The development of aneurysm was defined as an increase $\geq 50 \%$ relative to the original diameter of the aorta. ${ }^{A} P<0.01$, Student's $t$ test, compared with pretreatment. 


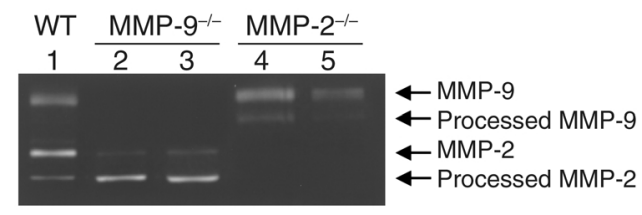

Figure 3

Gelatin zymography analysis of MMP activities expressed in experimental mice. Lane 1, WT; lanes 2 and 3, MMP-9KO; lanes 4 and 5, MMP-2KO.

$2.3 \pm 0.3)$ and media ( 1 week, $0.7 \pm 0.3 ; 2$ weeks, $1.0 \pm 0.0$ ) was not altered in MMP-2KO mice.

Effect of WT macrophage infusion on aneurysm formation in MMP-2KO and MMP-9KO mice. In human AAA tissue, the majority of MMP-2 is expressed by resident mesenchymal cells, although some macrophages also stain positively with MMP-2 antibodies (11). Macrophages are the primary source of MMP-9 in AAA tissue (21). To examine whether MMP-9 or MMP-2 from WT macrophages was adequate to reconstitute aneurysms in MMP-9KO or MMP-2KO mice, we injected MMP-9KO and MMP-2KO mice with CFSE-labeled peritoneal macrophages from their corresponding WT mice. This was done via a tail vein 1 day before $\mathrm{CaCl}_{2}$ aneurysm induction. After a week, mice were bled and white blood cells were isolated. FACScan analysis of white blood cells showed that 5\% of the white cells were CFSE-labeled positive. A second macrophage infusion was done 1 week after induction. MMP-9KO mice infused with MMP-9-producing peritoneal macrophages from WT mice developed significant arterial enlargement (Table 4; Figure 4a). A group of three MMP-9KO mice were sacrificed 1 week after WT macrophage infusion. Aortae were harvested, and aortic cells were isolated by enzymatic digestion. FACScan analysis of these cells at their nadir showed that $0.5 \%$ of aortic cells were CFSElabeled positive. Histologic analysis showed the disruption of elastic fibers in the media (Figure 4d). To insure that the aneurysm formation was not a nonspecific response to macrophage infusion, peritoneal macrophages harvested from MMP-9KO mice were infused into MMP-9 KO mice. There was no increase in aortic diameter (data not shown) or significant elastin destruction in mice infused with MMP-9KO macrophages (Figure 4c). WT macrophage infusion was not able to reconstitute the aneurysm in $\mathrm{CaCl}_{2}-$ treated MMP-2KO mice (Table 4), and the medial elastic lamellae were well preserved (Figure 4e).

To demonstrate that WT macrophages reach the aorta of the MMP-9KO mouse, MMP expressions in WT peritoneal macrophages and aortic tissues of MMP-9KO mice with WT peritoneal macrophage infusion were examined by zymography. MMP-9, but not MMP-2, expression was detected in isolated WT peritoneal macrophage (Figure 4b, lane 1). Both MMP-9 and MMP-2 were seen in the aortic tissues of MMP$9 \mathrm{KO}$ mice infused with WT peritoneal macrophages
(Figure 4b, lane 2). The presence of CFSE-labeled macrophages and MMP-9 in the aorta of MMP-9KO mice confirms the infiltration of intravenously infused WT macrophages. These results demonstrate that MMP-9 from macrophages and MMP-2 derived from mesenchymal cells work in concert to produce AAA.

\section{Discussion}

AAAs are characterized by structural alternations of the aortic wall resulting, in part, from degradation of the macromolecules, collagen and elastin. These changes are associated with an inflammatory infiltrate (4) and excessive production of MMPs $(8-11,22)$, which are assumed to orchestrate the widespread matrix destruction. Both serine and cysteine proteases are also present in diseased arterial tissue $(23,24)$. Further knowledge of which of these enzymes play an etiologic role in the process may lead to prospects for pharmacologic, rather than mechanical, treatment of AAA.

As with many chronic disease processes, descriptive human studies are enlightening but often fail to distinguish important causal factors from epiphenomena. Animal models are essential to precisely define to the mechanisms of aneurysm formation. Murine models are particularly useful because of the ability to produce and access a wide array of mice with targeted gene deletions. There are two commonly used, reliable models of AAA, both of which involve a local arterial injury followed by inflammatory cell invasion, increased expression of MMPs, matrix destruction, and a corresponding increase in vessel diameter. The elastase-induced aneurysm model involves isolation and infusion of a segment of the aorta with a pancreatic extract containing elastase. Done under superphysiologic pressures, this results in an immediate mechanical dilation followed by a second phase of more gradual expansion $(5,14)$. It is unclear precisely which enzymes in the porcine pancreatic extract cause the local inflammation, and variability in the preparation has led to inconsistencies in aneurysm formation (25). A second common aneurysm model was first described by Gertz et al. (6) in the rabbit carotid artery and subsequently used in the rabbit aorta (8). The local application of $\mathrm{CaCl}_{2}$ to the adventitial arterial surface induced an inflammatory response followed by aneurysmal dilation (8). We have used this technique to

\section{Table 3}

The concentration of macrophages in aorta of 129/SvEv and MMP$9 \mathrm{KO}$ mice

\begin{tabular}{lcccc}
\hline & Mouse strain & 1 week & 2 weeks & 10 weeks \\
Adventitia & $129 /$ SvEv & $3.0 \pm 0.0$ & $3.0 \pm 0.0$ & $1.0 \pm 0.0$ \\
& MMP-9KO & $2.0 \pm 0.6$ & $3.0 \pm 0.0$ & $2.3 \pm 1.2$ \\
\multirow{3}{*}{ Media } & $129 /$ SvEv & $2.0 \pm 1.0$ & $1.0 \pm 0.0$ & $0.5 \pm 0.7$ \\
& MMP-9KO & $1.7 \pm 1.7$ & $1.0 \pm 0.0$ & $1.3 \pm 1.2$
\end{tabular}

Macrophages in aortic adventitia and media were evaluated and scored with values from 0 to 3 . Zero indicates that there were no Mac3-positive cells, 3 indicates many cells in a limited area, and 1 and 2 are used to classify intermediate grades of infiltration. The values reflect the mean \pm SE. 


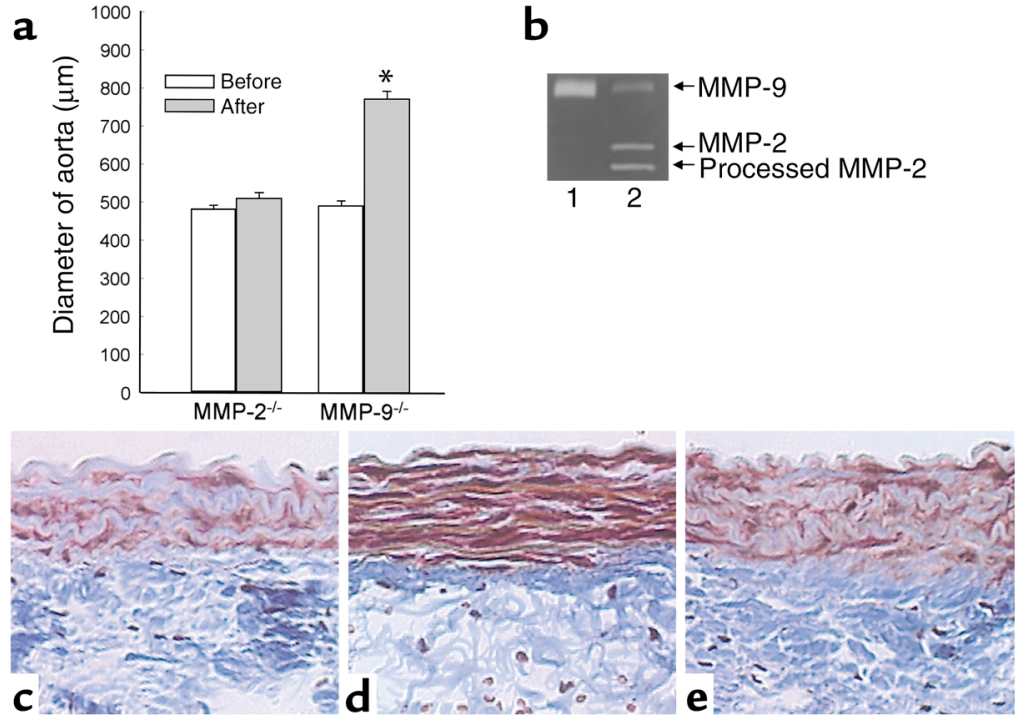

Figure 4

(a) Aortic diameter changes after $\mathrm{CaCl}_{2}$ treatment with WT macrophage infusion in MMP-2KO and MMP-9KO mice. Aortic diameters were measured before (white bars) and 8 weeks after (gray bars) $\mathrm{CaCl}_{2}$ incubation. Bars shown in each group represent the mean \pm SE of six to eight mice. The diameter in MMP-9KO mice increased significantly with macrophage infusion $\left({ }^{*} P<0.01\right)$. (b) Gelatin zymography analysis of MMP activities expressed in WT peritoneal macrophages (lane 1 ) and aorta of MMP$9 \mathrm{KO}$ mouse infused with WT macrophages (lane 2). (c-e) Histologic analysis. Paraffin sections were stained for elastin fibers (Trichrome), and sections were photographed and shown with the lumen at the top. (c) The MMP-9KO mouse infused with MMP-9KO macrophages; (d) the MMP-9KO mouse infused with WT macrophages; (e) the MMP-2KO mouse infused with WT macrophages.

generate a reproducible mouse model of aortic aneurysm disease (15) where the degree of dilatation is similar to that seen in the second phase (nonmechanical) of the elastase infusion model. Doxycycline, a nonspecific MMP inhibitor, attenuates aneurysm formation in a dose-dependent manner in this model (26).

The inflammatory infiltrate in human AAA tissue is spatially related to destruction of ECM macromolecules. The inflammatory infiltrate is thought to play an etiologic role in aneurysm formation by direct local production of matrix-degrading enzymes and production of cytokines that induce resident mesenchymal cell production of MMPs (27). Proinflammatory cytokines secreted from macrophages enhance MMP production by human vascular smooth muscle cells (27-29), and many of these cytokines are present within AAA tissue $(3,30)$. Our findings suggest that both the local mesenchymal cell expression and the macrophage expression of MMPs are required for aneurysm formation. Our data do not support the concept that MMP-2 is required for the processing of MMP-9, or the converse, since the processed form of both MMPs was seen in the zymograms from the alternate knockout mice. In fact, there appears to be increased activation of the remaining MMP, which was not sufficient to cause local injury or vessel dilation.

Pyo et al. (14) have shown that MMP-9 plays a pivotal role in aneurysm development in the elastase AAA infu- sion model. We tested the response of MMP-9KO mice using $\mathrm{CaCl}_{2}$ aneurysm induction. We also found that MMP9-deficient mice are resistant to aneurysm development. This was associated with preservation of the lamellar morphology of the aortic wall. D'Haes et al. (31) have reported decreased mobility of MMP-9-deficient macrophages in vitro, and Ducharme et al. (19) reported fewer tissue macrophages in MMP9-deficient mice 2 weeks after myocardial infarction. Therefore, we wanted to determine whether the absence of AAA formation in the MMP-9KO mice was due to decreased macrophage infiltration. Using macrophage-specific staining of aortic sections taken at 1,2, and 10 weeks after aneurysm induction, we performed an extensive examination of the aorta but could not identify a decrease in the number of aortic macrophages in MMP-9KO compared with WT mice. This finding is consistent with the observation of Pyo et al. (14) in the elastase infusion model. Macrophage infiltration in MMP-2KO mice was not impaired. MMP-2 deficiency had no effect on inflammatory cell invasion after allergic lung injury (20). Thus, the mechanism of protection from aneurysm development in MMP-9KO and MMP-2KO mice is related to the absence of local MMP-9 or MMP-2 productions rather than inability of macrophages to infiltrate to the site of injury. This is further supported by the observation of macrophages adjacent to intact elastic lamellae in the MMP-2KO and MMP-9KO mice.

Mesenchymal cells do not express MMP-9 under normal conditions, but smooth muscle cell production can be induced by a proinflammatory milieu, as occurs in AAA tissue (32). Conversely, macrophages produce

\section{Table 4}

Changes in aortic diameter in MMP-2KO and MMP-9KO mice with macrophage infusion

$\begin{array}{lcc} & \text { MMP-2KO } & \text { MMP-9KO } \\ \text { Treatment } & \mathrm{CaCl}_{2} & \mathrm{CaCl}_{2} \\ \text { Number } & 6 & 8 \\ \text { Pretreatment }(\mu \mathrm{m}) & 485 \pm 8.2 & 493 \pm 11.3 \\ \text { Post-treatment }(\mu \mathrm{m}) & 510 \pm 15.2 & 774 \pm 21.2^{\mathrm{A}} \\ \text { AAA development }(\%) & 0 & 88 \\ \text { Percent increase }(\%) & 7.4 & 57.3 \\ \text { Range of increase }(\%) & 0-9 & 46-83\end{array}$

Aortic diameters were measured before $\mathrm{CaCl}_{2}$ incubation (pretreatment) and at sacrifice (post-treatment). Measurements of aortic diameter are expressed as mean \pm SE. The percent increase is represented as compared with pretreatment. The development of aneurysm was defined as an increase of $\geq 50 \%$ relative to the original diameter of the aorta. ${ }^{A} P<0.01$, Student's $t$ test, compared with pretreatment. 
relatively large amounts of MMP-9, and this can be increased by proinflammatory cytokines (33). To confirm the requirement for macrophage-derived MMP-9 in AAA, we infused peritoneal macrophages from WT mice into MMP-9KO mice before aneurysm induction and 1 week later. This resulted in reconstitution of the aneurysm and damage to the elastin lamellae. Pyo et al. (14) have previously shown using sublethal irradiation and bone marrow transplantation that reconstitution of the MMP-9 genotype into the myelogenous cells of the MMP-9KO mice reconstituted aortic aneurysms. Our findings are somewhat more specific in demonstrating the importance of MMP-9 derived from macrophages alone. These findings in the murine model are consistent with histologic studies showing macrophages to be the primary source of MMP-9 in human AAA tissue $(9,11)$.

Among the MMPs, MMP-2 is unique in its ability to degrade both elastin and fibrillar collagen (34). It is found primarily in association with the mesenchymal cells of the media and adventitia, but small amounts may be produced by macrophages $(8,11)$. Our previous work has demonstrated that both total MMP-2 and activated MMP-2 are increased in AAA tissues (11). We hypothesized that mesenchymal cell MMP-2 was also involved in the initiation and progression of AAA. We used the MMP-2-deficient mice to test the effect of MMP-2 on aneurysm formation. Targeted deletion of MMP-2 also protected mice from aneurysm development after $\mathrm{CaCl}_{2}$ treatment. Histologically, the aorta of the MMP-2KO mice looks no different from the aorta of the corresponding WT mice treated with $\mathrm{NaCl}$. We believe that these findings are novel in demonstrating a key role for MMP-2 in the development of AAA. They are especially intriguing in that the presence of MMP-9 was unable to cause local tissue injury in the absence of MMP-2. To test whether protection from AAA in MMP- $2 \mathrm{KO}$ mice can be attributed to the MMP-2 deficiency in macrophages, we infused the WT peritoneal macrophages into MMP$2 \mathrm{KO}$ mice. By fluorescently labeling infused peritoneal macrophages, we were able to confirm that, at their nadir, they remained in the peripheral blood and aneurysm tissue 1 week after infusion. Unlike MMP$9 \mathrm{KO}$ mice, infusion of competent macrophages failed to produce aneurysms. This experiment showed that the required MMP-2 is supplied by other cells.

Since the knockout mice used in these studies were of different backgrounds, we cannot exclude the seemingly remote possibility that the requirements for MMP-9 or MMP-2 were specific to the genetic background of each strain. This seems particularly unlikely given the similarity of the aneurysm dilatation and histologic injury in these strains.

Previous studies have shown that both elastase and collagenase may be required for aneurysmal dilatation (35). Both fibrillar collagen and elastin are highly organized in the lamellar structure of the aortic media. One potential mechanism for the complementary role of
MMP-2 and MMP-9 is that MMP-2 is primarily acting as a collagenase initiating cleavage of the triple helix into one-quarter and three-quarter lengths (34). The single $\alpha$ chains could then be degraded by MMP-9, releasing the coiled elastin and causing it to become fattened and attenuated. The observations that MMP-2 and MMP-9 may work in concert to promote injury are supported by recent observations in a murine model of hepatitis (36). MMP-2 may be the primary interstitial collagenase in this murine AAA model.

These data demonstrate, for the first time to our knowledge, that a concerted role of MMP-2 and MMP-9 is required for aneurysmal degeneration and suggest that either or both could be targeted for the treatment of AAA. This study also suggests that an interaction is required between cellular products of invading macrophages and local mesenchymal cells. Understanding the relationship between MMPs secreted by invading macrophages and local mesenchymal cells has implications not only for AAA, but also for all other inflammatory diseases associated with organ injury from destruction of the ECM.

Note added in proof. Yu and Stamenkovic have shown that MMP-9 and MMP- 2 can activate TGF- $\beta$ by cleaving its latent form (37). Activation of TGF- $\beta$ plays an important role in the control of tissue remodeling in both physiological and pathological situations. On the other hand, both gelatinases are capable of proteolytically inactivating alpha-1-PI by cleaving serpins at an inhibitory site region. A recent study by Moraga et al. demonstrated that cleaved serpins induces expression of proinflammatory molecules in monocytes (38). Therefore, another potential mechanism for the complementary role of MMP-2 and MMP-9 could be through the activation of TGF- $\beta$ or inactivation (cleavage) of serpins.

\section{Acknowledgments}

This study was supported by NIH grant 5RO1HL62400-02 and American Heart Association grant $974015 \mathrm{~N}$ (B.T. Baxter).

1. Alcorn, H.G., Wolfson, S.K., Jr., Sutton-Tyrrell, K., Kuller, L.H., and O'Leary, D. 1996. Risk factors for abdominal aortic aneurysms in older adults enrolled in The Cardiovascular Health Study. Arterioscler. Thromb. Vasc. Biol. 16:963-970.

2. Ernst, C.B. 1993. Abdominal aortic aneurysm. N. Engl. J. Med. 328: $1167-1172$.

3. Pearce, W.H., and Koch, A.E. 1996. Cellular components and features of immune response in abdominal aortic aneurysms. Ann. NY Acad. Sci. 800: $175-185$

4. Bobryshev, Y.V., Lord, R.S., and Parsson, H. 1998. Immunophenotypic analysis of the aortic aneurysm wall suggests that vascular dendritic cells are involved in immune responses. Cardiovasc. Surg. 6:240-249.

5. Anidjar, S., et al. 1990. Elastase-induced experimental aneurysms in rats. Circulation. 82:973-981.

6. Gertz, S.D., Kurgan, S., and Eisenberg, D. 1988. Aneurysm of the rabbit common carotid artery induced by periarterial application of calcium chloride in vivo. J. Clin. Invest. 81:649-656.

7. Ricci, M.A., et al. 1996. Anti-CD 18 monoclonal antibody slows experimental aortic aneurysm expansion. J. Vasc. Surg. 23:301-307.

8. Freestone, T., et al. 1995. Inflammation and matrix metalloproteinases in the enlarging abdominal aortic aneurysm. Arterioscler. Thromb. Vasc. Biol. 15:1145-1151. 
9. Thompson, R.W., et al. 1995. Production and localization of 92-kilodalton gelatinase in abdominal aortic aneurysms. An elastolytic metalloproteinase expressed by aneurysm-infiltrating macrophages. J. Clin. Invest. 96:318-326.

10. Curci, J.A., Liao, S., Huffman, M.D., Shapiro, S.D., and Thompson, R.W. 1998. Expression and localization of macrophage elastase (matrix metalloproteinase-12) in abdominal aortic aneurysms. J. Clin. Invest. 102:1900-1910.

11. Davis, V.P.R., et al. 1998. Matrix metalloproteinase-2 production and its binding to the matrix are increased in abdominal aortic aneurysms. Arterioscler. Thromb. Vasc Biol. 18:1625-1633.

12. Petrinec, D., et al. 1996. Doxycycline inhibition of aneurysmal degeneration in an elastase-induced rat model of abdominal aortic aneurysm: preservation of aortic elastin associated with suppressed production of $92 \mathrm{kD}$ gelatinase. J. Vasc. Surg. 23:336-346.

13. Allaire, E., Forough, R., Clowes, M., Starcher, B., and Clowes, A.W. 1998. Local overexpression of TIMP-1 prevents aortic aneurysm degeneration and rupture in a rat model. J. Clin. Invest. 102:1413-1420.

14. Pyo, R., et al. 2000. Targeted gene disruption of matrix metalloproteinase-9 (gelatinase B) suppresses development of experimental abdominal aortic aneurysms. J. Clin. Invest. 105:1641-1649.

15. Longo, G.M., Pehm, J.P., Mayhan, W.G., and Baxter, B.T. 1999. Calciumchloride-induced experimental aneurysms in mice. Surg. Forum. L:450-451.

16. Itoh, T., et al. 1997. Unaltered secretion of beta-amyloid precursor protein in gelatinase A (matrix metalloproteinases 2)-deficient mice. J. Biol. Chem. 272:22389-22392.

17. Russell, H.K., Jr. 1972. A modification of Movat's pentachrome stain. Arch. Pathol. 94:187-191.

18. Goodall, S., Crowther, M., Hemingway, D.M., Bell, P.R., and Thompson, M.M. 2001. Ubiquitous elevation of matrix metalloproteinase- 2 expression in the vasculature of patients with abdominal aneurysms. Circulation. 104:304-309.

19. Ducharme, A., et al. 2000. Targeted deletion of matrix metalloproteinase-9 attenuates left ventricular enlargement and collagen accumulation after experimental myocardial infarction. J. Clin. Invest. 106:55-62.

20. Corry, D.B., et al. 2002. Decreased allergic lung inflammatory cell egression and increased susceptibility to asphyxiation in MMP2-deficiency. Nat. Immunol. 3:347-353.

21. Hibbs, M.S. 1992. Expression of $92 \mathrm{kDa}$ phagocyte gelatinase by inflammatory and connective tissue cells. Matrix Suppl. 1:51-57.

22. Newman, K.M., et al. 1994. Matrix metalloproteinases in abdominal aortic aneurysm: characterization, purification and their possible sources. Connect. Tissue Res. 30:265-276.
23. Rao, S.K., Reddy, K.V., and Cohen, J.R. 1996. Role of serine proteases in aneurysm development. Ann. NY Acad. Sci. 800:131-137.

24. Shi, G.P., et al. 1999. Cystatin C deficiency in human atherosclerosis and aortic aneurysms. J. Clin. Invest. 104:1191-1197.

25. Curci, J.A., and Thompson, R.W. 1999. Variable induction of experimental abdominal aortic aneurysms with different preparations of porcine pancreatic elastase. J. Vasc. Surg. 29:385.

26. Prall, A.K, et al. 2002. Doxycycline in patients with abdominal aortic aneurysms and in mice: comparison of serum levels and effect on aneurysm growth in mice. J. Vasc. Surg. 35:923-929.

27. Lee, E., et al. 1995. Human smooth muscle cell-monocyte interactions and metalloproteinase secretion in culture. Arterioscler. Thromb. Vasc. Biol. 15:2284-2289.

28. Keen, R.R., et al. 1994. Interleukin-1 beta induces differential gene expression in aortic smooth muscle cells. J. Vasc. Surg. 20:774-784.

29. Galis, Z.S., et al. 1994. Cytokine-stimulated human vascular smooth muscle cells synthesize a complement of enzymes required for extracellular matrix digestion. Circ. Res. 75:181-189.

30. Newman, K.M., Jean-Claude, J., Li, H., Ramey, W.G., and Tilson, M.D. 1994. Cytokines that activate proteolysis are increased in abdominal aortic aneurysms. Circulation. 90:11224-11227.

31. D'Haes, A., et al. 2000. In vivo neutrophil recruitment by granulocyte chemotactic protein- 2 is assisted by gelatinase B/MMP-9 in the mouse. J. Interferon Cytokine Res. 20:667-674.

32. Gurjar, M.V., Deleon, J., Sharma, R.V., and Bhalla, R.C. 2001. Role of reactive oxygen species in IL-1 beta-stimulated sustained ERK activation and MMP-9 induction. Am. J. Physiol. Heart Circ. Physiol. 281:H2568-H2574.

33. Welgus, H.G., et al. 1990. Neutral metalloproteinases produced by human mononuclear phagocytes. Enzyme profile, regulation, and expression during cellular development. J. Clin. Invest. 86:1496-1502.

34. Aimes, R., and Quigley, J. 1995. Matrix metalloproteinase-2 is an interstitial collagenase. J. Biol. Chem. 270:5872-5876.

35. Dobrin, P.B., and Mrkvicka, R. 1994. Failure of elastin or collagen as possible critical connective tissue alterations underlying aneurysmal dilatation. Cardiovasc. Surg. 2:484-488.

36. Wielockx, B., et al. 2001. Inhibition of matrix metalloproteinases blocks lethal hepatitis and apoptosis induced by tumor necrosis factor and allows safe antitumor therapy. Nat. Med. 7:1202-1208.

37. Yu, Q., and Stamenkovic, I. 2000. Cell surface-localized matrix metalloproteinase- 9 proteolytically activates TGF- $\beta$ and promotes tumor invasion and angiogenesis. Genes Dev. 14:163-176.

38. Moraga, F., Lindgren, S., and Janciaskiene, S. 2001. Effects of noninhibitory alpha-1-antitrypsin on primary human monocyte activation in vitro. Arch. Biochem. Biophys. 386:221-226. 